\title{
Effectiveness and Tolerability of Korean Red Ginseng Augmentation in Major Depressive Disorder Patients with Difficult-to-treat in Routine Practice
}

\author{
Kyung Ho Lee', Won-Myong Bahk², Soo-Jung Lee ${ }^{2}$, Chi-Un Pae ${ }^{2,3}$ \\ Departments of ${ }^{1}$ Dermatology and ${ }^{2}$ Psychiatry, ${ }^{3}$ Cell Death Disease Research Center, College of Medicine, The Catholic University of Korea, \\ Seoul, Korea
}

Objective: To test clinical effectiveness and tolerability of Korean Red Ginseng augmentation (RGA) in major depressive disorder (MDD) patients with difficult-to-treat.

Methods: Thirty six patients were enrolled in this 6 weeks, prospective, clinical trial. Rating scales were MontgomeryÅsberg Depression Rating Scale (MADRS), Patient Health Questionnaire-15, Clinical Global Impression-improvement (CGI-I), and Patient Satisfaction Score. The primary endpoint was a remission rate measured by MADRS score at the end of study $(\leq 10)$. Clinical outcomes and tolerability were assessed at baseline, week 2, and week 6 .

Results: Among 36 patients, 26 patients completed the study and 28 patients had post-baseline visit data. The remission rate by MADRS score was $39.3 \%(11 / 28)$ and $57.1 \%$ by CGI-I scores of 1 or 2 at the end of the study. The mean change of MADRS score was significantly decreased by $44.4 \%$ from baseline to the end of study. The most frequent adverse events were headache $(7 / 28,25.0 \%)$ during the study.

Conclusion: Our study indicates the putative effectiveness and tolerability of RGA for treating MDD with difficult-to-treat in clinical practice. However, adequately powered, randomized, controlled trials will be needed to confirm these results.

KEY WORDS: Therapeutics; Depressive disorder; Antidepressants; Red ginseng; Difficult-to-treat.

\section{INTRODUCTION}

A number of antidepressants have been developed and used to treat patients with major depressive disorders (MDD), but until now, the treatment of MDD has not been shown to be satisfactory with antidepressant treatment [1]. Indeed antidepressant monotherapy is a gold standard for MDD treatment, however, approximately 30$60 \%$ of MDD patients do not respond to antidepressant monotherapy in real practice [2-4].

According to the results from a recent large practical trial of MDD, the Sequenced Treatment Alternatives to Relieve Depression (STAR*D) study, the remission rate of

Received: May 25, 2020 / Revised: July 1, 2020

Accepted: July 7, 2020

Address for correspondence: Chi-Un Pae

Department of Psychiatry, Bucheon St. Mary's Hospital, College

of Medicine, The Catholic University of Korea, 327 Sosa-ro,

Wonmi-gu, Bucheon 14647, Korea

E-mail: pae@catholic.ac.kr

ORCID: https://orcid.org/0000-0003-1632-4248
MDD was $30 \%$ after the first drug treatment, $17 \%$ after the second level treatment, $14 \%$ after the third level treatment, and $13 \%$ after the fourth level treatment; and the cumulative remission rate following treatment for one year was only $70 \%$ [5]. Therefore, recent treatment guidelines for MDD includes switching to different antidepressant, combination of two or more antidepressants, or augmentation of psychotropics (i.g., thyroid hormone, lithium, tryptophan, psychostimulant, buspirone, atypical antipsychotics) as next level treatment option upon failure to current treatments $[6,7]$.

Interestingly, augmentation of aripiprazole which is one of atypical antipsychotic for treating psychotic disorders has been widely utilized for MDD patients with the extended understanding of the action mechanism of the drug today $[8,9]$. When it comes to Red Ginseng (RG), it contains a large amount of saponins, and ginsenosides which are $\operatorname{Rg} 1, \operatorname{Rg} 2$, and $\operatorname{Rg} 3$ as one of these saponins. RG has known to regulate oxidative stress, apoptotic re-

(c) This is an Open-Access article distributed under the terms of the Creative Commons Attribution Non-Commercial License (http://creativecommons.org/licenses/by-nc/4.0) which permits unrestricted non-commercial use, distribution, and reproduction in any medium, provided the original work is properly cited. 
sponses, and pro-inflammatory mediators, which are considered etiological perspectives for development of MDD [10]. RG has also many other promising neuropsychopharmacological effects on development and treatment for MDD including activating BDNF signaling pathway and up-regulation of hippocampal neurogenesis, promotion of the neurite outgrowth via ERK and AKT dependent signaling pathways $[11,12]$, modulation of $5-\mathrm{HT}_{2 \mathrm{~A}}$ receptors [13], increasing hippocampal 5-HT level [14], modulation of dopaminergic/noradrenergic neuron by protection of inflammatory reaction [15], inhibition of microglial cell, regulation of HPA axis [16], and enhancement of neuronal cell survival [17] and increased expression of BDNF/CREB [18] and so on [10]. Therefore $\mathrm{RG}$ is known to have positive effects on improving cognitive function, regulation of mood, accelerating mental/motor function, stress relief, and recovering physical energy. Such effects of RG should be beneficial for MDD patients since those symptoms are common in MDD patients [19]. However, there has been a dearth of evidence from clinical trials till today, particularly for MDD patients with difficult-to-treat. Hence the present study was conducted to test the clinical effectiveness and tolerability of Korean RG augmentation (RGA) in difficult-to-treat MDD patients who were currently on antidepressant monotherapy.

\section{METHODS}

The present study was a prospective, open label, 6-week study to evaluate the effectiveness and tolerability of RGA in difficult-to-treat MDD patients who were currently on antidepressant monotherapy. Effectiveness and tolerability were assessed at baseline (week 0), week 2, and week 6 .

Diagnosis was based on the Diagnostic and Statistical Manual of Mental Disorders 5th edition criteria [20]. Included subjects were on 3 or more on Clinical Global Impression-Improvement (CGI-I) score despite of current antidepressant therapy with proper dosage (based on drug label information) and duration (at least 6 weeks) along with at least one or more previous failure history to antidepressant treatment. Patients were excluded if they were pregnant or nursing. Those who have current other Axis I/II diagnoses other than MDD as well as unstable medical or neurological disorders were also excluded. Those who have any psychotic symptomatology and formal/brief psy- chotherapeutic treatment were excluded as well.

Clinical outcome scales were Montgomery-Åsberg Depression Rating Scale (MADRS) [21], Patient Health Questionnaire-15 (PHQ-15) [22], CGI-I [23], and Patient Satisfaction Score (PSS) [24]. The primary endpoint was the remission rate measured by MADRS score $(\leq 10)$ at the end of study. The secondary endpoints were the response rate defined as a $50 \%$ or more reduction in the MADRS score and the mean changes of MADRS, PHQ-15, and PSS scores from baseline to the end of study.

The current antidepressant and other psychotropics at the time of enrollment were maintained throughout the study. Korean Red Ginseng extract was administrated to subjects in capsule form. Aside from ginseng extract, capsules contained ginsenosides, such as $\operatorname{Rg} 1(3.4 \mathrm{mg} / \mathrm{g}), \mathrm{Rb} 1$ (7.25 mg/g), Rg3s $(0.23 \mathrm{mg} / \mathrm{g})$, Re (2.42 mg/g), Rc (2.9 $\mathrm{mg} / \mathrm{g}), \mathrm{Rb} 2$ (2.75 mg/g), Rd (0.64 mg/g), Rf (1 mg/g), Rh1 $(0.17 \mathrm{mg} / \mathrm{g}), \mathrm{Rg} 2 \mathrm{~s}(0.32 \mathrm{mg} / \mathrm{g})$, and other minor ginsenosides. The dosage of RG was $2 \mathrm{~g} /$ day based on the previous study [25].

Statistical analysis was performed using NCSS 2007 and PASS 2008 (NCSS LLC, Kaysville, UT, USA). The data was analyzed by modified intent-to-treat population with the last observation carried forward for end of treatment analysis. Non-parametric analyses were performed because of the small sample size. $95 \%$ confidence intervals (Cl) obtained where appropriate. The descriptive statistics and Wilcoxon Signed Rank test were performed where appropriate, and statistical significance was determined at $p<0.05$. Under a two-tailed alpha value of 0.05 , the power of our sample to detect an effect size $(\mathrm{d}=0.5)$ was $80 \%$, which corresponds to a difference in the MADRS of approximately 9 points.

The study was approved by the Institutional Review Board at Bucheon St. Mary's hospital and was conducted in compliance with the Declaration of Helsinki (IRB approval number. HC12HISI0102). All patients provided informed consent.

\section{RESULTS}

Among 36 patients, 26 patients completed the study and 28 patients had post-baseline visit data. Ten (27.8\%) patients terminated the study early as a result of adverse events $(n=1,2.8 \%)$, protocol violation $(n=1,2.8 \%)$ and lost to follow up ( $n=8,22.2 \%)$. Twenty-six $(72.2 \%)$ of the 
patients completed the full 6-week trial, $28(77.8 \%)$ of the patients returned for at least one post follow-up visit.

The mean age of the patients was approximately 48 years and three fourths was female. One had a history of admission and approximately one in five had a family history of MDD. The baseline MADRS, CGI-S, PHQ-15 and PSS scores were 21.8, 4.2, 7.7 and 4.6, respectively. The

Table 1. Baseline characteristic of samples

\begin{tabular}{lc}
\hline \multicolumn{1}{c}{ Parameters } & Values \\
\hline Age (yr) & $47.8 \pm 14.4$ \\
Onset age (yr) & $43.9 \pm 13.7$ \\
Weight (kg) & $59.7 \pm 10.6$ \\
Number of previous failure to antidepressant therapy & $2.5 \pm 1.1$ \\
CGl-S & $4.2 \pm 0.6$ \\
PSS & $4.7 \pm 0.9$ \\
MADRS & $21.6 \pm 4.9$ \\
PHQ-15 & $6.9 \pm 4.4$ \\
Sex (female) & $27(75.0)$ \\
Religion (yes) & $13(36.1)$ \\
Job & $4(11.1)$ \\
Marital status (married) & $21(58.3)$ \\
Family history & $6(16.7)$ \\
Medical comorbidity, past & $28(77.8)$ \\
Current medical comorbidity & $18(50.0)$ \\
Current antidepressants & \\
Duloxetine & $2(5.6)$ \\
Venlafaxine & $9(25.0)$ \\
Paroxetine & $15(41.7)$ \\
Sertraline & $2(5.6)$ \\
Fluoxetine & $2(5.6)$ \\
Escitalopram & $3(8.3)$ \\
Mirtazapine & $3(8.3)$ \\
\hline Values are & \\
\hline
\end{tabular}

Values are presented as mean \pm standard deviation or number (\%). CGI-S, Clinical Global Impression-severity; PSS, Patient Satisfaction Score; MADRS, Montgomery-Åsberg Depression Rating Scale; PHQ-15, Patient Health Questionnaire-15. most commonly used current antidepressant was paroxetine and followed by venlafaxine. Table 1 summarizes the clinical and demographic data of the study participants.

The remission rate by MADRS score was $39.3 \%(11 / 28)$ and $57.1 \%$ by CGI-I scores of 1 or 2 at the end of the study. The response rate by MADRS score was $42.9 \%$ $(12 / 28)$ at the end of study (Table 2). The mean changes of MADRS, PSS and PHQ-15 scores was significantly improved by $44.4 \%$ ( $p<0.001$ ), 31.9\% ( $p<0.001)$ and $26.1 \%(p=0.019)$, respectively from baseline to the end of study (Table 2).

The most frequent adverse event was headache (7/28, $25.0 \%)$ and followed by depression $(6 / 28,21.4 \%)$, fatigue $(6 / 28,21.4 \%)$ and anxiety $(5 / 28,17.9 \%)$ during the study. Table 3 presents the summary of AEs during the study.

\section{DISCUSSION}

The present study showed the effectiveness and toler-

Table 3. Incidence of adverse events during the study $(n=28)$

\begin{tabular}{ll}
\hline \multicolumn{1}{c}{ Parameters } & Values \\
\hline Headache & $7(25)$ \\
Depression & $6(21.4)$ \\
Fatigue & $6(21.4)$ \\
Anxiety & $5(17.9)$ \\
Somnolence & $3(10.7)$ \\
Pharyngitis & $1(3.6)$ \\
Gastric discomfort & $1(3.6)$ \\
Low energy & $1(3.6)$ \\
Binge eating & $1(3.6)$ \\
Palpitation & $1(3.6)$
\end{tabular}

Values are presented as number (\%).

Table 2. Clinical outcome summary during the study $(n=28)$

\begin{tabular}{|c|c|c|c|c|c|}
\hline Parameters & Baseline & Week 2 & Week 6 & Statistical values* & $95 \%$ confidence interval ${ }^{* *}$ \\
\hline MADRS & $21.6 \pm 4.9$ & $17.1 \pm 5.1$ & $12.0 \pm 6.2$ & $Z=-4.629, p<0.001$ & \\
\hline PSS & $4.7 \pm 0.9$ & $5.8 \pm 1.0$ & $6.9 \pm 1.2$ & $Z=-4.345, p<0.001$ & \\
\hline PHQ-15 & $6.9 \pm 4.4$ & $5.6 \pm 3.7$ & $5.1 \pm 4.3$ & $Z=-2.339, p=0.019$ & \\
\hline \multicolumn{6}{|l|}{ Remission rate } \\
\hline MADRS & & 1 (3.6) & $11(39.3)$ & & $0.2213-0.5927$ \\
\hline CGI-I (1 or 2$)$ & & $3(10.7)$ & $16(57.1)$ & & $0.3743-0.7497$ \\
\hline \multicolumn{6}{|l|}{ Response rate } \\
\hline MADRS & & $1(3.6)$ & $12(42.9)$ & & $0.2503-0.6257$ \\
\hline
\end{tabular}

Values are presented as mean \pm standard deviation or number $(\%)$.

MADRS, Montgomery-Åsberg Depression Rating Scale; PSS, Patient Satisfaction Score; PHQ-15, Patient Health Questionnaire-15; CGI-S, Clinical Global Impression-severity.

*Comparison between Baseline and end of the study and Wilcoxon Signed Rank test. ${ }^{* *} 95 \% \mathrm{Cl}$ confidence interval (with continuity correction) at the end of study. 
ability of RGA in difficult-to-treat MDD patients who were currently on antidepressant monotherapy.

The potential antidepressant-like effects of RG were continuously replicated in many basic researches and few clinical trials for depressive and related disorders. A plausible mechanism for RGA to antidepressants might be its modulation and regulation on different neurotransmitters, neuroendocrine pathways, neuronal signaling pathways, neuro-inflammatory pathways and neuroprotection process and so on [10]. In fact, stress and depression impair the expression and function of BDNF in hippocampus and prefrontal cortex, which is a critical element in synaptic plasticity $[11,26]$. RG restores the signaling pathways of BDNF in a positive direction in MDD patients. $\mathrm{Rb} 1$ has an effects of modulation on $5-\mathrm{HT}_{2 \mathrm{~A}}$ receptors leading to increase of 5-HT in synapse [13]. In addition, saponin has reversing effects on chronic mild stress-induced decrease of monoamine neurotransmitters including norepinephrine, dopamine, and homovanillic acid [27]. Depression is known to have abnormal cytokine homeostasis, the activation of proinflammatory cytokines and the suppression of anti-inflammatory cytokines [28]. It was found that Rg1 stimulated nitric oxide and proinflammatory cytokines (IL-1 beta, IL-6, and TNF-alpha), while Rb1 showed an antagonistic activity on them [29]. $\mathrm{Rb} 1$ and $\mathrm{Rg} 1$ also regulate the genetic expression of bcl-2 and bax, both of which have been implicated in neuronal toxicity and degeneration [29]. RG improved anhedonia, hopelessness and improved sleep disruption through the modulation levels of corticosterone, testosterone, androgen receptor, and glucocorticoid receptor [16], it was also found to counteract the rise in corticotropin-releasing factor, adrenocorticotropic hormone, and cortisol in animal model study [30]. Neuroprotective effects of RG through modulation of antioxidant signal pathways and effects of autonomic system stabilization have been also continuously found $[17,31]$.

In the first double-blind, randomized clinical trial $(\mathrm{n}=$ 63) [31], RG had shown better cognitive response and stabilization of sympathetic nervous system through regulation of epinephrine and steroid, which is in line with the positive effects of GR on stress control and also findings from a remote study (improvement of anxiety, depression, fatigue and somatic symptoms proven by a decrease in cortisol/DHEA-S ratio). In addition, a recent small study [32] has also shown the clinical effects of RG on improve- ment of residual depressive symptoms and somatic complaints, expanding treatment option for such patients. Standardized ginseng extract has also shown significant improvement of working memory performance [33].

The main strength of the present study is that our findings are the first one to have shown a possibility of RGA as a potential alternative treatment option for difficult-to-treat MDD patients. According to the results, RGA achieved remission and response rates of approximately $39 \%$ and $43 \%$ which are in line with other trials investigating augmentation treatments $[9,34]$. This finding is comparable to those from STAR*D trial data; indeed, remission was only 20 to $30 \%$ at level 1 and 2 treatment step, however, it decreased to 10 to $20 \%$ at level 3 and 4 treatment step. Thus, our remission rate is approximately $20 \%$ better than that from STAR*D trial, regardless of treatment level. However, the remission and response rates were quite disappointing and lower than those reported from other augmentation trials, possibly explained by no early treatment effects of RG, pharmacological characteristics of $R G$, low sensitivity of rating scale to detect difference with herbal medications such as RG, and sample characteristics. The PSS scores was also significantly decreased indicating an easy acceptance by patients themselves, which is an intriguing finding since patient-preference for treatment is one of crucial factors for better compliance and clinical outcomes in depression [35]. In addition, somatic symptoms were also significantly improved as evidenced in decrease of PHQ-15 score, which is potentially supporting the RG effects on multiple physical complaints commonly seen in MDD patients. This is in line with the beneficial effects of RG on homeostasis of autonomic system as reported in previous studies $[16,25,31]$. Finally RGA was also found to be tolerable in the present study; only one patient was found to withdraw the study due to $\mathrm{AE}$ and all $\mathrm{AEs}$ were mild-to-moderate.

The present study has clear limitations. The study design was not a randomized, double blind, placebo-controlled method, which might lead to potential selection bias and could not exclude the natural improvement. The sample size was small even though the power was adequate to detect symptomatic change throughout the study; however, the possibility of false positive should be considered. The pharmacokinetics of the RG was not monitored. Finally, the follow-up period was relatively short. Hence, the period over which the effect was main- 
tained could not be determined.

Our study indicates the putative effectiveness and tolerability of RGA for treating MDD with difficult-to-treat in routine practice. However, adequately powered, randomized, controlled trials will be needed to confirm these results.

\section{Acknowledgments}

The red ginseng was provided by Korea Ginseng Corp.

\section{Conflicts of Interest}

No potential conflict of interest relevant to this article was reported.

\section{Author Contributions}

Conceptualization: Chi-Un Pae. Data acquisition: Chi-Un Pae. Formal analysis: Chi-Un Pae and Kyung Ho Lee. Intellectual comments: Won-Myong Bahk and Soo-Jung Lee. Draft writing: Chi-Un Pae and Kyung Ho Lee. Editing: Chi-Un Pae, Kyung Ho Lee, Won-Myong Bahk, and Soo-Jung Lee.

\section{ORCID}

Kyung Ho Lee

https://orcid.org/0000-0003-1473-2125

Won-Myong Bahk https://orcid.org/0000-0002-0156-2510

Soo-Jung Lee

https://orcid.org/0000-0002-1299-5266

Chi-Un Pae

https://orcid.org/0000-0003-1632-4248

\section{REFERENCES}

1. Han C, Wang SM, Bahk WM, Lee SJ, Patkar AA, Masand PS, et al. A pharmacogenomic-based antidepressant treatment for patients with major depressive disorder: results from an 8-week, randomized, single-blinded clinical trial. Clin Psychopharmacol Neurosci 2018;16:469-480.

2. Serretti A. The present and future of precision medicine in psychiatry: focus on clinical psychopharmacology of antidepressants. Clin Psychopharmacol Neurosci 2018;16:1-6.

3. Gaynes BN, Warden D, Trivedi MH, Wisniewski SR, Fava M, Rush AJ. What did STAR*D teach us? Results from a largescale, practical, clinical trial for patients with depression. Psychiatr Serv 2009;60:1439-1445.

4. Fabbri C, Serretti A. Genetics of treatment outcomes in major depressive disorder: present and future. Clin Psychopharmacol Neurosci 2020;18:1-9.

5. Rush AJ. STAR*D: what have we learned? Am J Psychiatry 2007; 164:201-204.

6. Seo SJ, Bahk WM, Wang HR, Woo YS, Park YM, Jeong JH, et al. Korean Medication Algorithm for depressive disorders
2017: Third revision. Clin Psychopharmacol Neurosci 2018; 16:67-87.

7. Kennedy SH, Lam RW, McIntyre RS, Tourjman SV, Bhat V, Blier $\mathrm{P}$, et al. Canadian Network for Mood and Anxiety Treatments (CANMAT) 2016 clinical guidelines for the management of adults with major depressive disorder: Section 3. Pharmacological Treatments. Can J Psychiatry 2016;61:540-560.

8. Pae CU, Serretti A, Patkar AA, Masand PS. Aripiprazole in the treatment of depressive and anxiety disorders: a review of current evidence. CNS Drugs 2008;22:367-388.

9. Han C, Wang SM, Bahk WM, Lee SJ, Patkar AA, Masand PS, et al. The potential utility of aripiprazole augmentation for major depressive disorder with mixed features specifier: a retrospective study. Clin Psychopharmacol Neurosci 2019;17: 495-502.

10. Hou W, Wang Y, Zheng P, Cui R. Effects of ginseng on neurological disorders. Front Cell Neurosci 2020;14:55.

11. Xu D, Wang C, Zhao W, Gao S, Cui Z. Antidepressant-like effects of ginsenoside Rg5 in mice: involving of hippocampus BDNF signaling pathway. Neurosci Lett 2017;645:97-105.

12. Jiang B, Xiong Z, Yang J, Wang W, Wang Y, Hu ZL, et al. Antidepressant-like effects of ginsenoside Rg1 are due to activation of the BDNF signalling pathway and neurogenesis in the hippocampus. Br J Pharmacol 2012;166:1872-1887.

13. Yamada $\mathrm{N}$, Araki $\mathrm{H}$, Yoshimura $\mathrm{H}$. Identification of antidepressant-like ingredients in ginseng root (Panax ginseng C.A. Meyer) using a menopausal depressive-like state in female mice: participation of 5-HT2A receptors. Psychopharmacology (Berl) 2011;216:589-599.

14. Jang D, Lee HJ, Lee K, Kim KR, Won R, Lee SE, et al. White ginseng ameliorates depressive behavior and increases hippocampal 5-HT level in the stressed ovariectomized rats. Biomed Res Int 2019;2019:5705232.

15. Wang GL, He ZM, Zhu HY, Gao YG, Zhao Y, Yang H, et al. Involvement of serotonergic, noradrenergic and dopaminergic systems in the antidepressant-like effect of ginsenoside Rb1, a major active ingredient of Panax ginseng C.A. Meyer. J Ethnopharmacol 2017;204:118-124.

16. Mou Z, Huang Q, Chu SF, Zhang MJ, Hu JF, Chen NH, et al. Antidepressive effects of ginsenoside $\mathrm{Rg} 1$ via regulation of HPA and HPG axis. Biomed Pharmacother 2017;92:962-971.

17. Dong X, Zheng L, Lu S, Yang Y. Neuroprotective effects of pretreatment of ginsenoside $R b 1$ on severe cerebral ischemia-induced injuries in aged mice: involvement of anti-oxidant signaling. Geriatr Gerontol Int 2017;17:338-345.

18. Kim C, Lee JH, Baek SH, Ko JH, Nam D, Ahn KS. Korean red ginseng extract enhances the anticancer effects of sorafenib through abrogation of CREB and c-Jun activation in renal cell carcinoma. Phytother Res 2017;31:1078-1089.

19. Lin IM, Fan SY, Yen CF, Yeh YC, Tang TC, Huang MF, et al. Heart rate variability biofeedback increased autonomic activation and improved symptoms of depression and insomnia among patients with major depression disorder. Clin Psycho- 
pharmacol Neurosci 2019;17:222-232.

20. American Psychiatric Association. Diagnostic and statistical manual of mental disorders: DSM-5. 5th ed. Arlington:American Psychiatric Association;2013.

21. Montgomery SA, Asberg M. A new depression scale designed to be sensitive to change. Br J Psychiatry 1979;134:382-389.

22. Han C, Pae CU, Patkar AA, Masand PS, Kim KW, Joe SH, et al. Psychometric properties of the Patient Health Questionnaire-15 (PHQ-15) for measuring the somatic symptoms of psychiatric outpatients. Psychosomatics 2009;50:580-585.

23. Guy W. ECDEU assessment manual for psychopharmacology. Rockville:U.S. Dept. of Health, Education, and Welfare, Public Health Service, Alcohol, Drug Abuse, and Mental Health Administration, National Institute of Mental Health, Psychopharmacology Research Branch, Division of Extramural Research Programs; 1976.

24. Chapman CR, Casey KL, Dubner R, Foley KM, Gracely RH, Reading AE. Pain measurement: an overview. Pain 1985;22: 1-31.

25. Shin WY, Kang EH, Kim KJ, Song YJ, Yu BH. Effect of Korean red ginseng on stress responses and beta-adrenergic receptor function in a normal population. Korean J psychopharmacol 2009;20:135-140.

26. Ryu S, Jeon H, Koo S, Kim S. Korean red ginseng enhances neurogenesis in the subventricular zone of 1-methyl-4-phenyl1,2,3,6-tetrahydropyridine-treated mice. Front Aging Neurosci 2018;10:355.

27. Dang $\mathrm{H}$, Chen Y, Liu X, Wang Q, Wang L, Jia W, et al. Antidepressant effects of ginseng total saponins in the forced swimming test and chronic mild stress models of depression. Prog Neuropsychopharmacol Biol Psychiatry 2009;33:14171424.

28. Petralia MC, Mazzon E, Fagone $\mathrm{P}$, Basile MS, Lenzo V,
Quattropani MC, et al. The cytokine network in the pathogenesis of major depressive disorder. Close to translation? Autoimmun Rev 2020;19:102504.

29. Joo SS, Won TJ, Lee DI. Reciprocal activity of ginsenosides in the production of proinflammatory repertoire, and their potential roles in neuroprotection in vivo. Planta Med 2005;71: 476-481.

30. Jiang N, Zhang BY, Dong LM, Lv JW, Lu C, Wang Q, et al. Antidepressant effects of dammarane sapogenins in chronic unpredictable mild stress-induced depressive mice. Phytother Res 2018;32:1023-1029.

31. Baek JH, Heo JY, Fava M, Mischoulon D, Choi KW, Na EJ, et al. Effect of Korean red ginseng in individuals exposed to high stress levels: a 6-week, double-blind, randomized, placebo-controlled trial. J Ginseng Res 2019;43:402-407.

32. Jeong $\mathrm{HG}$, Ko YH, Oh SY, Han C, Kim T, Joe SH. Effect of Korean red ginseng as an adjuvant treatment for women with residual symptoms of major depression. Asia Pac Psychiatry 2015;7:330-336.

33. Scholey A, Ossoukhova A, Owen L, Ibarra A, Pipingas A, He $\mathrm{K}$, et al. Effects of American ginseng (Panax quinquefolius) on neurocognitive function: an acute, randomised, double-blind, placebo-controlled, crossover study. Psychopharmacology (Berl) 2010;212:345-356.

34. Han C, Wang SM, Kwak KP, Won WY, Lee H, Chang CM, et al. Aripiprazole augmentation versus antidepressant switching for patients with major depressive disorder: a 6-week, randomized, rater-blinded, prospective study. J Psychiatr Res 2015;66-67:84-94.

35. Granger AL, Fehnel SE, Hogue SL, Bennett L, Edin HM. An assessment of patient preference and adherence to treatment with Wellbutrin SR: a web-based survey. J Affect Disord 2006;90:217-221. 